\title{
Engineering quantum materials with chiral optical cavities
}

\author{
Hannes Hübener, ${ }^{1, *}$ Umberto De Giovannini, ${ }^{1, \dagger}$ Christian Schäfer, ${ }^{1}$ Johan \\ Andberger ${ }^{2}$ Michael Ruggenthaler, ${ }^{1}$ Jerome Faist, ${ }^{2}$ and Angel Rubio ${ }^{1,3, \ddagger}$ \\ ${ }^{1}$ Max Planck Institute for the Structure and Dynamics of Matter and \\ Center for Free Electron Laser Science, 22761 Hamburg, Germany \\ ${ }^{2}$ ETH Zürich, Institute of Quantum Electronics, Auguste-Piccard-Hof 1, Zürich 8093, Switzerland \\ ${ }^{3}$ Center for Computational Quantum Physics (CCQ), \\ The Flatiron Institute, 162 Fifth avenue, New York NY 10010.
}

\begin{abstract}
Strong light-matter coupling in quantum cavities provides a pathway to break fundamental materials symmetries, like time-reversal symmetry in chiral cavities. This Comment discusses the possibility to realize non-equilibrium states of matter that have so far been only accessible in ultrafast and ultrastrong laser driven materials.
\end{abstract}

Recent developments at the interface between quantum materials and photonics are opening alternative avenues for materials design. On the one hand are materials with emergent properties that are determined by the effects of quantum mechanics on a macroscopic scale so called, quantum materials [1]. They can yield novel functionalities [2] and are considered as a route towards engineering materials properties on demand [3]. On the other hand we have matter interacting with light. Driving materials out of equilibrium has been the most widely sought route to access hidden phases of matter, that is to say states of matter that are not thermally accessible, but can be exposed by symmetry breaking excitations. Light driven states can also be designed to transiently manipulate the crystal structure and symmetry $[4,5]$ or to form light-matter hybrid states with new physical properties [6-9]. In the latter case, light is not just a mere spectator that perturbatively acts on the material to extract information about its properties but is itself a part of the non-equilibrium state. This brings materials science and photonics together where in turn also the symmetries of light are being engineered through its interaction with solids.

In the strong light-matter coupling regime the quantum state of the material is no longer only determined by the symmetry of the crystal and its electronic structure. With light becoming a constituent component of the new state its symmetry properties become as relevant as that of the equilibrium material and can even be viewed as a new, synthetic material dimension [10], that arise from reinterpreting degrees of freedom as additional spatial dimension. Such strong light-matter interactions leading to hybrid light-matter states have long been investigated in the context of quantum optics where specifically tailored quantum cavities have been used to enhance the coupling [11]. Thus the two fields of ultrafast laser driven materials and quantum photonics are converging to a point where material properties of light-

\footnotetext{
* hannes.huebener@mpsd.mpg.de

$\dagger$ umberto.de-giovannini@mpsd.mpg.de

‡ angel.rubio@mpsd.mpg.de
}

driven phases can be controlled through the symmetry of light via the enhanced interaction provided by optical cavities.

The idea is to equip the fields in the cavity with properties such that one can control the resulting hybrid states and especially to design them to break specific symmetries in the material. One of the most consequential symmetries for materials is time-reversal symmetry. It is well known that a magnetic field breaks the time-reversal symmetry of charged particles in materials, that is to say electrons or nuclei, because it affects them differently depending on the sign of their momentum. Similarly, a circularly polarized light field affects charges with opposing momenta in opposite ways. As a result the dynamics and possibly the properties of materials can be fundamentally different when such a time-reversal symmetry breaking field is dressing the electronic structure. Here, optical cavities can be especially important, because they allow the coupling of a material to quantum fluctuations of the vacuum, thereby creating a new groundstate and hence a new material. So before we discuss the design of lightmatter states in more general terms it is worthwhile to first consider how one can create particular symmetries of photons that could be imprinted in the matter and especially how to design chiral electromagnetic modes in optical cavities. The chirality can have either spatial character, where the polarization follows for instance a corkscrew through space, or it can be of temporal character, where the polarization rotates in a plane like the hands of a clock. Only the latter results in breaking of time-reversal symmetry inside the cavity.

In Fig. 1 different examples of simple symmetry breaking cavity modes are shown. A normal (Fabry-Perot) cavity, (Fig. 1 a), formed by two parallel mirrors breaks neither the spatial inversion nor the time-reversal symmetry and consists of two orthogonal linearly polarized modes. A helical mode, such as the one depicted in Fig. $1 \mathrm{~b}$ ), can arise in a cavity with specifically designed metamaterial mirrors, that, in contrast to "normal" mirrors, will reflect a right-handed polarized light into light with the same handedness [12]. In such a mode, the electric field follows a helix in space but is locally linearly polarized at all points. As a result, one can only expect 

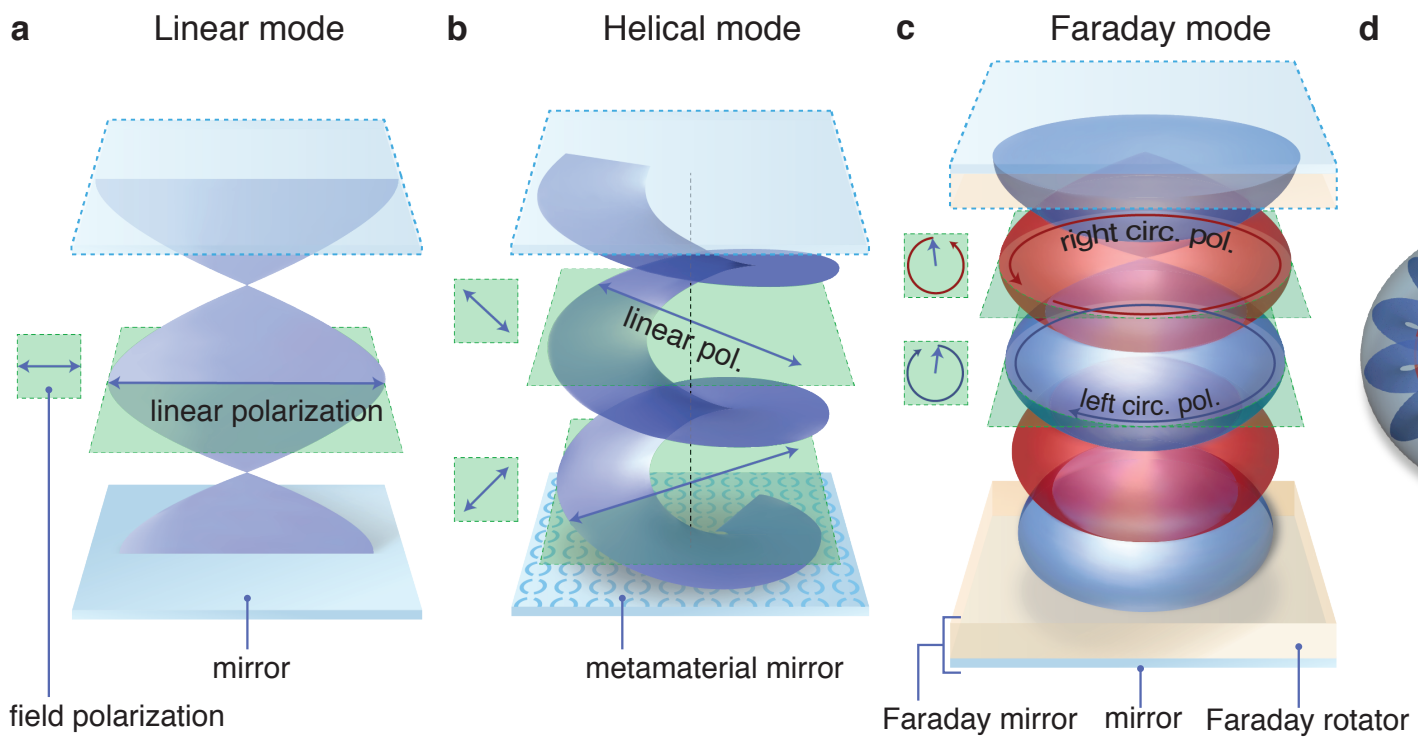

Ring cavity

spatial

temporal

spatial and temporal

chiral modes

FIG. 1. Cavity modes for different cavity environments leading to symmetry breaking configurations.. The electromagnetic field forms standing waves in a cavity that, depending on the boundary conditions, can break spatial, temporal or spatio-temporal symmetries. a, a mode (dark blue shape) of a linearly polarized cavity with conventional mirrors shown as light blue planes. $\mathbf{b}$, if the mirrors only reflect light with either left or right circular polarization the cavity mode attains a helical, or corkscrew, structure, where in each horizontal plane the field is linearly polarized, but rotates in the vertical direction (see the projection of the polarisation vector on the green plains cutting the cavity standing wave). This can be achieved by employing structured, metamaterial mirrors. c, a possible realization of time-reversal symmetry breaking cavity modes is offered by the use of Faraday mirrors, composed of a normal mirror (light blue) and Faraday rotator (beige), where, due to Faraday reflection, two standing waves of opposite circular polarization (shown in red and blue respectively with their polarisation in the green planes is highlighted on the green insets) form with alternating nodal structure. d, alternatively, a cavity with a ring geometry can be designed to sustain a running wave with a chosen circular polarization by means of an handedness filter, shown as a golden section, realizable with a combination of a Faraday rotator and polarization optics.

quantum states that arise from breaking spatial symmetries to occur in such cavities. Fig. $1 \mathrm{c}$ ) presents an example of the standing wave mode of a cavity that displays time-reversal symmetry breaking. One should first note that, since the two orthogonally polarized modes of a planar Fabry-Perot cavity are degenerate in frequency, such a chiral mode can be excited through an external circularly polarized drive in a normal cavity. However, to design new materials the time-reversal symmetry should be lifted by the cavity itself which can be achieved using so-called Faraday mirrors that use a magneto-optical effect to lift the degeneracy between the left and right polarised modes (red and blue standing waves in Fig. 1c) with opposite rotation direction. Another way of achieving a cavity that breaks time-reversal symmetry is by using a ring configuration where the handedness of the circular polarized mode is selected by the combination of a Faraday rotator and polarization optics, as shown in Fig. $1 \mathrm{~d}$ ). In both cases, the very interesting conceptual aspect of these cavities is that the symmetry is "imprinted" onto the material inserted inside the cavity from the Faraday rotator by the way of vacuum field fluc- tuations. Designing and fabricating cavities that break time-reversal symmetry and therefore sustain the mode schematically described in Fig. 1 c) and d) while being suitable for solid-state applications raises a special set of challenges that we address at the end of this article.

But what are the novel properties that one can create and how can we design the cavity to host novel quantum states? A possible route to answer this question can be found when considering the theoretical description of hybrid light-matter states. It will become clear next that we can infer some characteristics of the dressing in cavities from the comparison between the Floquet and the quantum quantum electrodynamics (QED) formulation of light-matter dressing. We will discuss briefly this materials engineering idea and come back later to the chiral cavities to show how the cavity facilitates the realisation of those states without the need of external driving.

In fact, describing the physical properties that arise from the interaction of light and matter in terms of, so called, dressed states, has been widely pursued in quantum optics and materials science. This description leads for strong coupling to the concept of hybrid 


\section{Box 1: Floquet and quantum light-matter dressing at a glance}

Floquet materials engineering. The Floquet formalism decomposes the dynamics of a periodically driven system into static states that are harmonics of the driving frequency $\omega$ and constant phases $E_{\alpha}$ with the ansatz

$$
\left|\Psi_{\alpha}(t)\right\rangle=e^{-i E_{\alpha} t} \sum_{m=-\infty}^{\infty} e^{i m \omega t}\left|\psi_{m}^{\alpha}\right\rangle
$$

The static states $\psi_{m}^{\alpha}$ are the components of the dressed state for the harmonic $m$. In the basis of harmonics the time-dependent Schrödinger equation reads

$$
\begin{aligned}
\left\{E_{\alpha}-m \omega-\hat{H}_{0}\right\} & \psi_{m}^{\alpha}(\mathbf{r}) \\
& =A_{0} \boldsymbol{\epsilon} \cdot \boldsymbol{\nabla}\left(\psi_{m-1}^{\alpha}(\mathbf{r})-\psi_{m+1}^{\alpha}(\mathbf{r})\right) \\
& -\frac{1}{2} A_{0}^{2}\left(\psi_{m-2}^{\alpha}(\mathbf{r})+\psi_{m+2}^{\alpha}(\mathbf{r})\right)
\end{aligned}
$$

which can be visualized as the matrix structure:

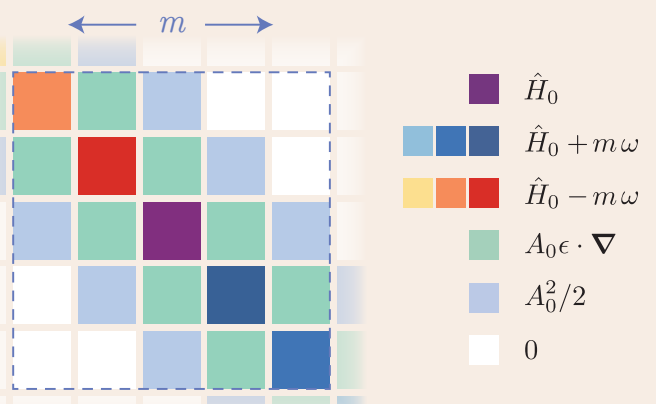

where each block represents an operator in real space. The diagonal blocks are composed from the groundstate Hamiltonian and multiples of the driving frequency $H_{0}+$ $m \omega(m=\ldots,-2 \square,-1 \square, 0 \square, 1 \square, 2 \square \ldots)$, while the off-diagonal blocks determine the coupling between harmonics through the classical field, represented by the vector potential amplitude $A_{0}$ and the polarization vector $\epsilon$. Coupling. The light-matter coupling strength is determined by the amplitude of the externally applied monochromatic electric field: $A_{0}=-E / \omega$.

The dashed lines indicate that the matrix extends beyond the blocks that are shown. Here we use atomic units.
QED materials engineering. The fundamental theory for the description of light-matter coupling is quantumelectrodynamics (QED), where both light and matter are described in terms of quantized particles. The coupled electron-photon state can be written as a dressed state

$$
\left|\Psi_{\alpha}\right\rangle=\sum_{m=0}^{\infty}|m\rangle\left|\psi_{m}^{\alpha}\right\rangle
$$

where $|m\rangle$ are coherently shifted Fock number states [22], $\psi_{m}^{\alpha}$ the electronic components. In the basis of these number states, the QED Hamiltonian reads

$$
\begin{aligned}
& \left\{E_{\alpha}-\left(\omega+A_{0}^{2}\right)\left(m+\frac{1}{2}\right)-\hat{H}_{0}\right\} \psi_{m}^{\alpha}(\mathbf{r}) \\
& =A_{0} \boldsymbol{\epsilon} \cdot \boldsymbol{\nabla}\left(\sqrt{m} \psi_{m-1}^{\alpha}(\mathbf{r})-\sqrt{m+1} \psi_{m+1}^{\alpha}(\mathbf{r})\right) \\
& -\frac{1}{2} A_{0}^{2}\left(\sqrt{m} \sqrt{m-1} \psi_{m-2}^{\alpha}(\mathbf{r})+\sqrt{m+1} \sqrt{m+2} \psi_{m+2}^{\alpha}(\mathbf{r})\right)
\end{aligned}
$$

wich can be visualized as the matrix structure:

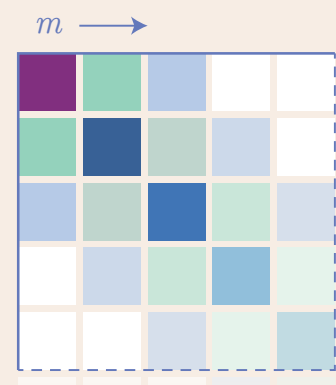

$$
\begin{aligned}
& \hat{H}_{0} \\
& \hat{H}_{0}+m\left(\omega+A_{0}^{2}\right) \\
& A_{0} \epsilon \cdot \nabla \sqrt{m+1} \\
& A_{0}^{2} \sqrt{m+1} \sqrt{m+2} / 2
\end{aligned}
$$$$
0
$$

where each block represents an operator in real space. Similar to the Floquet case the diagonal blocks consist of the uncoupled electronic Hamiltonian and replica energies $\hat{H}_{0}+m\left(\omega+A_{0}^{2}\right)(m=0 \square, 1 \square, 2 \square, 3,4 \square \ldots)$, where we have omitted the constant shift $\left(\omega+A_{0}^{2}\right) / 2$. The off-diagonals control the coupling in the same form as in the Floquet case, with the only difference of a scaling by $\sqrt{m+1}(m=0 \square, 1 \square, 2-, 3 \ldots)$.

Coupling. The light-matter coupling strength is determined by the geometry of the cavity. e.g. $A_{0}=$ $\sqrt{4 \pi /(\omega V)}$, where $V$ is the volume of the cavity and $\omega$ the mode energy. light-matter quasiparticles that can modify the behavior of the material completely, especially considering their ability to attain collective ordering and condensate into a new phase (for instance Bose condensation of exciton polaritons, c.f. references in [13]). The dressed quasiparticle picture emerges in different theoretical approaches for describing quantum light-matter interaction, of which two are shown in Box 1. Floquet theory recasts the time-dependent Schrödinger equation with a classical, monochromatic (laser) field into a static eigenvalue problem for dressed states. The periodicity in time leads to a periodic quasi-energy structure in analogy to the periodic momentum space structure resulting from a periodic lattice. Remarkably, almost the same eigenvalue problem arises, when applying a dressed ansatz to non-relativistic $\mathrm{QED}$, the fundamental quantum theory of light-matter interaction, in the non-relativistic regime [14]. As illustrated in Box 1, both theories result in correlated electron-photon states that obey very similar Hamiltonians. The main difference between the two is that QED has a groundstate and thus is bound from below, while Floquet theory, describing a driven state, has an un- 
bounded spectrum with both positive and negative photon replicas. However, it shows the close connection between driven quantum states and those forming in materials in optical cavities.

But how can a driven state be similar to an equilibrium state? Indeed, the idea to use external lasers to control and stabilize excitations in materials on a fast time-scale has been recently developed into a field that is sometimes referred to as, Floquet engineering $[8,9]$. It is based on the tenet that the dressed quantum states of the material under continuously applied, oscillating perturbation are stationary and described by the Hamiltonian in Box 1 . These states are then thought to behave analogously to groundstate energy levels but endowing the material with different properties and thus can guide our conception of which cavity dressed equilibrium states can be created for different symmetry-tailored cavity modes.

The driven-equilibrium Floquet state, however, is hard to realize in materials, because a variety of processes, such as dissipation, decoherence, impurity scattering and excited state lifetimes limit strongly the realization and survival of the Floquet states one is interested in. Furthermore, while the Floquet dressing results in states with altered properties, the actual population of these states is specific to the laser-driving protocol, which complicates the selective preparation of a specific Floquet state. The analogy between Floquet and cavity QED light-matter hybridization shown in Box 1 provides a possible way to mitigate these problems by using the cavity photon modes to dress the electronic states such that the dressing takes place on the level of single photons and is governed by QED. Controlling the nature of the quantum vacuum surrounding a material inside a resonating cavity can therefore have an effect on the properties of this material even without any external driving and imprint the symmetry properties of (chiral) cavity photons onto the electronic structure.

One can however also turn this point of view on its head. The physical processes behind dressed states by laser driving and by coupling to quantum photons in a cavity are quite different, however the properties of such dressed states can be very similar, since they follow very similar Hamiltonians. This sole idea sets up a general framework for the design of novel quantum materials in cavities based on what is known about driven systems: if the transient non-equilibrium phase can be cast in terms of dressed states, it can be potentially realized as quasiequilibrium or groundstates in optical cavities. Floquet theory here provides the blueprints of what are the required parameters (mode polarisation symmetry, energy, population, etc.) a cavity should have to achieve the desired dressed states. In other words, this point of view allows to translate the striking properties of the Floquet phases induced by, for example, chiral light [8, 9] into properties of an equilibrium material in a chiral cavity.

We can call this new way to conceive the design of materials cavity QED materials engineering. The spirit of the possibilities that such a scheme entails is sketched in Fig. 2 and we will now turn to discuss some of them showing the central role that chiral cavities can play in this context.

There are already several theoretical proposals to design quantum material properties by cavity dressing that do not necessarily rely on chiral photons or any other specific symmetry breaking induced by the cavity mode. For example, just to mention a few, cavity enhanced transition temperature in superconductivity [15], enhancement of the superconducting gap [16], cavity mediated control of electron-phonon coupling [17] and electron-photon pairing [18] as a promising routes towards cavity controlled superconducting materials, cavity-mediated superradiance [19], and tuning of excitonic states in layered materials [20]. Moreover, recent experiments on two-dimensional materials embedded in dielectric microcavities [21-23] have shown that the collective character of dressed states reduces the scattering with disorder stabilizing valley polarization at possibly room temperature with implications for valleytronics [24] and photovoltaics. An interesting avenue is the stabilisation of a new groundstate by laser driving that has recently been demonstrated for the paraelectric-ferroelectric transition in $\mathrm{SrTiO}_{3}[4,5]$, which can in principle, be realised in a cavity by coupling the relevant order parameter of the transition to the cavity mode [25, 26] showing the possibility that light-dressing can change a material down to the lattice structure.

The potential of chiral cavity dressing can be inferred from the many intriguing properties of dressed systems with broken time-reversal symmetry that have been discussed in the context of Floquet engineering. The most noted results are proposals related to the manipulation of topological properties of materials and hence the control of transport properties, especially the anomalous (spin) Hall conductance and their protected edge states, higher order topological states, fractional Chern insulators and unconventional topological superconductors, among many other proposals. To take a specific example, it was shown both theoretically [27] and experimentally [28] that graphene can experience a light-induced anomalous Hall effect upon irradiation by chiral light [29] which corresponds to the design of the celebrated Haldane Hamiltonian by driving.

While for graphene, the Floquet-Chern insulator has been shown to be challenging to realize, for a chiral cavity dressed graphene the expected topological changes should occur in the groundstate of the system (Ref. 30 already discusses this for a driven cavity). In a chiral cavity such effect could be interpreted as having the electrons in the graphene feeling the effect of the magnetic field in the Faraday rotator through the exchange of virtual chiral cavity photons. Similarly, one can aim at designing three dimensional Dirac and Weyl materials. Breaking of time-reversal symmetry by the chiral cavity modes moves the Weyl points in the Brillouin zone proportional to the strength of the light matter cavity coupling, that is to say light-mode volume, and one can manipulate such 


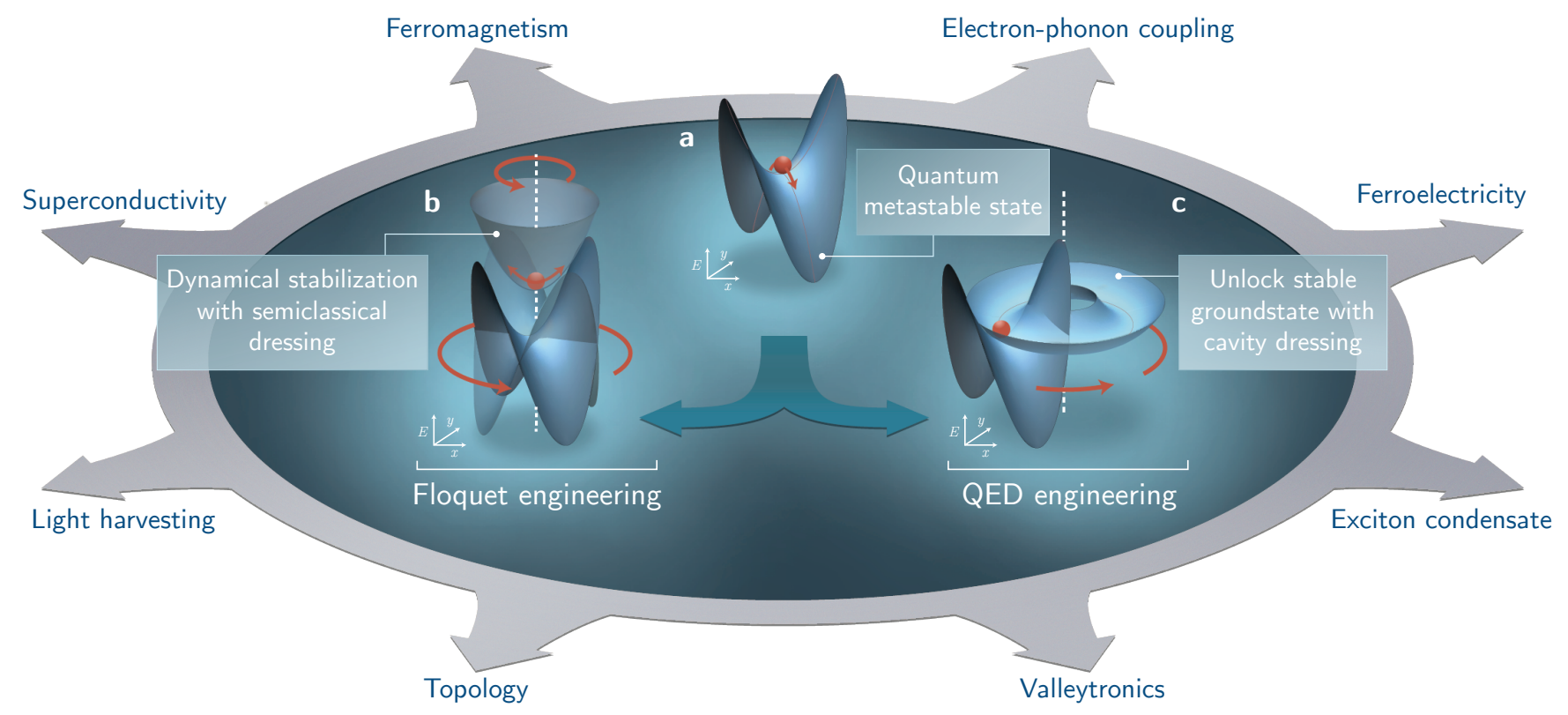

FIG. 2. QED materials engineering and phenomenology. a, a saddle potential is a typical example a quantum metastable state. b, in Floquet engineering such a state can be stabilized by moving it fast enough with oscillating fields shown as a rotating saddle point potential. This mechanism has been exploited in experiments, leading to phase transitions in the non-equilibrium state, such as light driven superconductivity, ferroelectricity, magnetism and topology among others. c, alternatively, material states can be hybridized with a cavity photon endowing the system with a new, stable groundstate. Such new states can have similar properties as the driven non-equilibrium phases, opening a new avenue for materials property design. The axes labels $x$ and $y$ indicate spatial dimensions and $E$ the potential.

materials by selectively bringing opposing Weyl point pairs together and thus cancel their Berry curvature or by splitting three dimensional Dirac Fermions into their Weyl Fermion constituents [31]. The Berry curvature dipole [32], that contributes to non-linear responses of the current and optical probes in inversion symmetry broken systems can be manipulated to enhance or reduce the non-linear properties by chiral cavity photons.

This short sketch of what lies ahead of us in this new field leaves many questions open that can only be answered through detailed experimental and theoretical investigations. This includes practical challenges, like the realization of time-reversal breaking cavities, but also many theoretical and fundamental questions.

When comparing the semi-classical Floquet and cavity QED dressing it is important to keep in mind that classical fields couple to the electronic structure of a material in proportion to their intensity and consequently a strong dressing effect requires a strong field. The vacuum of resonating cavities, instead, couples to the material via quantum fluctuations of the cavity modes and we can get strong field physics in the cavity without the presence of an external field. The effective light matter coupling cannot anymore be scaled with the laser power but is determined by the cavity geometry. In a very simple approximation, one may see this light-matter coupling as being proportional to the vacuum zero point energy of the light in the cavity. As the latter is constant and equal to half a light quanta, the vacuum electric field is then inversely proportional to the square root of the cavity volume. Indeed, the requirement of extremely small mode volume - necessary for the enhancement of vacuum fluctuations - precludes the use of free-space optics for either the cavity or the Faraday mirrors. The strength of interaction is however not solely defined by the cavity but moreover amplified by the amount of charged particles, that is to say density of polarizable material. As a consequence, antenna- and circuit-based approaches using a combination of high conductivity metals and materials with strong gyromagnetic response such as graphene or narrow-gap semiconductors are definitely promising ways to attack the problem, since they, in principle, allow the whole structure to keep highly subwavelength dimensions. Indeed, terahertz metamaterials coupled to cyclotron resonances in two-dimensional electron gases have enabled the exploration of the so-called ultrastrong light-matter coupling, regime where the vacuum Rabi frequency is comparable to the one of the bare electronic resonance [33]. However, the metamaterial used in these studies have displayed linearly polarized resonances in their near field. To achieve one of the main goals proposed in this article of breaking time-reversal symmetry by using chiral photons, it is then necessary to design new resonators that would exhibit similar light-matter coupling strengths but for circularly polarized light in their near-field. As a general strategy, such a structure 
could be designed based on two LC resonators or resonant antenna perpendicular to each other. This approach should enable coupling strength comparable to the ones achieved in linearly polarized metamaterials, and such cavities could be considered as the metamaterial equivalent of the Faraday cavity shown schematically in Fig. 1c. By capacitively or inductively coupling two such metametarial structures, one could even consider reproducing the ring geometry shown in Fig. 1d.

Theoretical challenges are also many. For instance, what we illustrated here applies to perfect, closed quantum cavities. However, to increase the light-matter coupling and to access the properties of the material in a cavity, in practice one has to create an open quantum system which affects the lifetime of the equilibrium state (or condensate) that can be formed inside it and also dictates the performance of the material. In this context of materials design we have to understand what happens when we externally pump the cavity. So once we have changed the groundstate of a system, which properties does this new state have? Similarly, our theoretical understanding of cavity dressing is mainly based on the assumption that the confined photon field can be described with the long-wavelength approximation, that is to say there is no spatial effect from the field. What can be achieved by going beyond this assumption is still largely unexplored. Furthermore, with the success of realizing topological Fermions in materials and the possibility to engineer their behavior through cavities, we might ask the question if optical cavities can serve as platforms for further solid-state realization of concepts from high-energy physics, such as broken parity-time $(P T)$ symmetry.

Despite these challenges for realizing cavity dressed materials both in conventional and in particular in chiral cavities, this Comment aims at providing a perspective on their potential and inspire work towards the realization of these systems. Especially for chiral cavities, the analogy drawn to non-equilibirum Floquet phases promises to be particularly productive, because of the consequences of breaking materials symmetries for applications in future quantum technologies.

\section{ACKNOWLEDGEMENTS}

We are grateful to Shunsuke A. Sato, Dongbin Shin, Michael A. Sentef, Enrico Ronca, Simone Latini, Dmitri Basov, Jean-Marc Triscone, Abhay Pasupathy, Eugene Demler, Andrea Cavalleri, Atac Imamoglu, Johannes Flick, Antoine Georges and Andrew Millis for the fruitful discussion. We acknowledge financial support from the European Research Council(ERC-2015-AdG-694097) and the Cluster of Excellence Advanced Imaging of Matter (AIM) EXC 2056 - 390715994. The Flatiron Institute is a division of the Simons Foundation. Support by the Max Planck Institute - New York City Center for NonEquilibrium Quantum Phenomena is acknowledged. HH and UDG contributed equally to this work.

\section{BIBLIOGRAPHY}

[1] Keimer, B. \& Moore, J. E. The physics of quantum materials. Nat. Phys. 13, 1045-1055 (2017).

[2] Tokura, Y., Kawasaki, M. \& Nagaosa, N. Emergent functions of quantum materials. Nat. Phys. 13, 1056-1068 (2017).

[3] Hsieh, D., Basov, D. N. \& Averitt, R. D. Towards properties on demand in quantum materials. Nat. Mater. 16, 1077-1088 (2017).

[4] Nova, T. F., Disa, A. S., Fechner, M. \& Cavalleri, A. Metastable ferroelectricity in optically strained SrTiO3. Science 364, 1075-1079 (2019).

[5] Li, X. et al. Terahertz field-induced ferroelectricity in quantum paraelectric SrTiO 3. Science 364, 1079-1082 (2019).

[6] Lindner, N. H., Refael, G. \& Galitski, V. Floquet topological insulator in semiconductor quantum wells. Nat. Phys. 7, 490-495 (2011).

[7] Wang, Y. H., Steinberg, H., Jarillo-Herrero, P. \& Gedik, N. Observation of Floquet-Bloch States on the Surface of a Topological Insulator. Science 342, 453-457 (2013).

[8] Oka, T. \& Kitamura, S. Floquet Engineering of Quantum Materials. Annu. Rev. Condens. Matter Phys. 10, 387408 (2018).

[9] Rudner, M. S. \& Lindner, N. H. Band structure engineering and non-equilibrium dynamics in Floquet topological insulators. Nat. Rev. Phys. 2, 229-244 (2020).

[10] Ozawa, T. \& Price, H. M. Topological quantum matter in synthetic dimensions. Nat. Rev. Phys. 1, 349-357 (2019).

[11] Kockum, A. F., Miranowicz, A., De Liberato, S., Savasta, S. \& Nori, F. Ultrastrong coupling between light and matter. Nat. Rev. Phys. 1, 19-40 (2019).

[12] Plum, E. \& Zheludev, N. I. Chiral mirrors. Appl. Phys. Lett. 106, 221901 (2015).

[13] Kasprzak, J. et al. Bose-Einstein condensation of exciton polaritons. Nature 443, 409-414 (2006).

[14] Schafer, C., Ruggenthaler, M. \& Rubio, A. Phys. Rev. A 98, 043801 (2018).

[15] Thomas, A. et al. Exploring Superconductivity under Strong Coupling with the Vacuum Electromagnetic Field . ArXiv e-prints (2019). 1911.01459.

[16] Curtis, J. B., Raines, Z. M., Allocca, A. A., Hafezi, M. \& Galitski, V. M. Cavity Quantum Eliashberg Enhancement of Superconductivity. Phys. Rev. Lett. 122, 167002 (2019).

[17] Sentef, M. A., Ruggenthaler, M. \& Rubio, A. Cavity quantum-electrodynamical polaritonically enhanced electron-phonon coupling and its influence on superconductivity. Sci. Adv. 4, eaau6969 (2018).

[18] Schlawin, F., Cavalleri, A. \& Jaksch, D. Cavity-Mediated Electron-Photon Superconductivity. Phys. Rev. Lett. 122, 133602 (2019).

[19] Mazza, G. \& Georges, A. Superradiant Quantum Materials. Phys. Rev. Lett. 122, 017401 (2019).

[20] Latini, S., Ronca, E., De Giovannini, U., Hübener, H. \& Rubio, A. Cavity Control of Excitons in TwoDimensional Materials. Nano Lett. 19, 3473-3479 (2019).

[21] Chen, Y.-J., Cain, J. D., Stanev, T. K., Dravid, V. P. \& Stern, N. P. Valley-polarized exciton-polaritons in a monolayer semiconductor. Nat. Photon. 11, 431 (2017). 
[22] Sun, Z. et al. Optical control of room-temperature valley polaritons. Nat. Photon. 11, 491 (2017).

[23] Dufferwiel, S. et al. Valley-addressable polaritons in atomically thin semiconductors. Nat. Photon. 11, 497 (2017).

[24] Vitale, S. A. et al. Valleytronics: Opportunities, Challenges, and Paths Forward. Small 14, 1801483 (2018).

[25] Ashida, Y. et al. Quantum Electrodynamic Control of Matter: Cavity-Enhanced Ferroelectric Phase Transition. ArXiv e-prints (2020). 2003.13695.

[26] Latini, S. et al. Cavity induced ferroelectric phase transition in $\overline{\mathrm{SrTiO}}_{3}$. (to be publihsed) (2020).

[27] Oka, T. \& Aoki, H. Photovoltaic Hall effect in graphene. Phys. Rev. B 79, 081406 (2009).

[28] McIver, J. W. et al. Light-induced anomalous Hall effect in graphene. Nat. Phys. 16, 1-4 (2019).

[29] Sato, S. A. et al. Microscopic theory for the light-induced anomalous $\overline{\text { Hall }}$ effect in graphene. Phys. Rev. B 99, 214302 (2019).

[30] Wang, X., Ronca, E. \& Sentef, M. A. Cavity quantum electrodynamical Chern insulator: Towards lightinduced quantized anomalous Hall effect in graphene. Phys. Rev. B 99, 235156 (2019).

[31] Hübener, H., Sentef, M. A., De Giovannini, U., Kemper, A. F. \& Rubio, A. Creating stable FloquetWeyl semimetals by laser-driving of 3D Dirac materials. Nat. Commun. 8, 13940 (2017).

[32] Sodemann, I. \& Fu, L. Quantum Nonlinear Hall Effect Induced by Berry Curvature Dipole in Time-Reversal Invariant Materials. Phys. Rev. Lett. 115, 216806 (2015).

[33] Scalari, G. et al. Ultrastrong Coupling of the Cyclotron Transition of a 2D Electron Gas to a $\mathrm{THz}$ Metamaterial. Science 335, 1323-1326 (2012). 